\title{
Serum ferritin is correlated with non-alcoholic fatty liver disease in middle-aged and older patients with type 2 diabetes
}

\author{
Jun-Xin Yan ${ }^{1}$, Bin-Jing Pan' ${ }^{1}$ Ping-Ping Zhao ${ }^{1}$, Li-Ting Wang ${ }^{1,2}$, Jing-Fang Liu ${ }^{1,2}$ and Song-Bo Fu ${ }^{1,2}$ \\ ${ }^{1}$ The First Clinical Medical College, Lanzhou University, Lanzhou, Gansu, China \\ ${ }^{2}$ Department of Endocrinology, The First Hospital of Lanzhou University, Lanzhou, Gansu, China \\ Correspondence should be addressed to J-F Liu: ljf824168@126.com
}

\begin{abstract}
Objective: Previous studies have shown the correlations between serum ferritin and non-alcoholic fatty liver disease (NAFLD) or diabetes. However, this relationship remains unclear in patients with type 2 diabetes (T2DM) with NAFLD. Therefore, this study aimed to elaborate the relationship between serum ferritin levels and NAFLD in middle-aged and older patients with T2DM and further explored the biomarkers for NAFLD in T2DM. Methods: A total of 805 middle-aged and older patients with T2DM were divided into NAFLD and non-NAFLD groups, and their serum ferritin levels were compared. Next, NAFLD group were divided into five subgroups according to the quintile levels of serum ferritin, and the differences in the constituent ratios of NAFLD were analyzed. A logistic regression analysis was performed to determine the risk factors for NAFLD in patients with T2DM.

Results: The serum ferritin levels were significantly higher in T2DM patients with NAFLD $(168.47(103.78,248.00) \mathrm{ng} / \mathrm{mL})$ than in the non-NAFLD patients $(121.19(76.97,208.39)$ $\mathrm{ng} / \mathrm{mL}$ ). The constituent ratios of NAFLD were significantly higher in the F5 and F4 groups than in the $\mathrm{F} 2$ or $\mathrm{F} 1$ groups (22.70 and $22.70 \%$ vs. 15.90 and $16.90 \%$, respectively; $P<0.05)$. Binary logistic regression analysis showed that serum ferritin $(P=0.001)$ was an independent risk factor for NAFLD in patients with T2DM.

Conclusions: Serum ferritin levels were significantly increased in T2DM with NAFLD, and the constituent ratios of NAFLD increased gradually along with the increased levels of serum ferritin. Thus, serum ferritin is an independent risk factor for NAFLD in patients with T2DM.

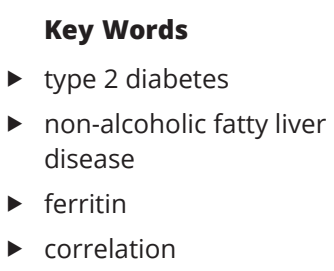

Endocrine Connections (2021) 10, 1560-1569
\end{abstract}

\section{Introduction}

Non-alcoholic fatty liver disease (NAFLD), which is the excessive deposition of fat ( $>5 \%$ of the liver weight) in liver cells without causes such as heavy drinking, hypothyroidism, or drugs, is considered a liver manifestation of metabolic syndrome (MS), or metabolic dysfunction-associated fatty liver disease. NAFLD is one of the most common chronic liver diseases in the world. The report estimated the prevalence of NAFLD is $25 \%$ globally, 23\% in Europe, the highest in the Middle East and South America, and the lowest in Africa (1). NAFLD prevalence varies in most Asian countries; in China, it ranges from 12.5 to $38 \%$ (2).

Studies have shown that changes in the prevalence of NAFLD in recent years are parallel to the prevalence of obesity and MS. NAFLD is closely related to type 2 diabetes (T2DM), both of which have the pathophysiological manifestations of excessive fat accumulation and insulin resistance $(3,4,5)$.

Approximately $10 \%$ of adults have diabetes and $90 \%$ of whom have T2DM (6). The overall prevalence of NAFLD

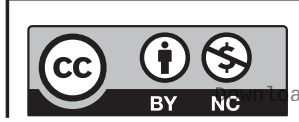

This work is licensed under a Creative Commons Attribution-NonCommercial 4.0 International License. ded from Bioscientifica.com at 04/26/2023 12:26:51PM 
in patients with $\mathrm{T} 2 \mathrm{DM}$ is $55.5 \%$, which is more than two times in the general population (7). In recent years, many researchers have stated that T2DM is one of the important risk factors for NAFLD (8). The typical insulin resistance and obesity-mediated oxidative stress and inflammation in patients with T2DM lead to the stress state and dysfunction of liver mitochondria, which furthers the development of NAFLD (9). In addition, liver biopsy revealed that steatohepatitis represented the sole feature of liver damage in T2DM. Therefore, NAFLD generally presents as nonalcoholic steatohepatitis (NASH) in patients with T2DM. NASH may be one of the early complications of T2DM due to its pathophysiological correlation with insulin resistance (10).

Serum ferritin is an important indicator of iron storage in the body. As a strong oxidant, iron can promote the oxidative stress process of hyperglycemia to pancreatic beta cells (11). Studies have found that serum ferritin is associated with increased blood glucose, retinopathy, nephropathy, and vascular dysfunction in patients with T2DM (12). The changes in serum ferritin levels may also be associated with the occurrence of NAFLD in patients with T2DM (13). Iron overload may affect glucose and lipid metabolism in the pancreatic beta cells and in the liver, muscles, and adipose tissue (14). Iron can generate reactive oxygen species (15), which directly damages the DNA, lipids, and proteins in liver cells through the consumption of ATP and glutathione (16). There is also evidence that iron regulates adipose tissue inflammation, adiponectin levels, and adipose tissue lipolysis and that iron in adipose tissue may promote the progression of NAFLD by mediating insulin resistance (17).

Studies have also shown that serum ferritin may be involved in the occurrence of fatty liver as an inflammatory cytokine, but the specific source of higher serum ferritin level remains unclear. One source may be the release of stored iron or the inflammatory state of the body (18). Ryan et al. believed that the iron in the liver was the main source of serum ferritin and that serum ferritin was the main prognostic factor for NAFLD (19). Therefore, this study aimed to analyze the correlations between serum ferritin levels and NAFLD in middle-aged and older patients with T2DM in order to further explore a new biological marker for the diagnosis and treatment of T2DM patients with NAFLD.

\section{Materials and methods}

\section{Study population}

A total of 1213 patients with T2DM admitted to the Department of Endocrinology of Lanzhou University First
Hospital from 2016 to 2020 were recruited. After screening according to inclusion and exclusion criteria, 805 middleaged and older patients with T2DM (490 men and 315 women) were enrolled in the study.

This study was approved by the Ethics Committee of the First Hospital of Lanzhou University, and written informed consent was obtained from all of the participants. The inclusion and exclusion criteria for this study were as follows.

Inclusion criteria: we included patients with T2DM aged $\geq 45$ years and those with complete clinical data.

Exclusion criteria: we excluded patients with malignant tumors, acute infection, hemochromatosis, chronic inflammatory diseases, acute inflammation of the liver, elevated liver enzymes due to unknown causes, iron deficiency anemia, acute and chronic blood loss, excessive drinking (weekly alcohol intake $>140 \mathrm{~g}$ for men and $>70 \mathrm{~g}$ for women), drug-induced hepatitis, autoimmune hepatitis, viral hepatitis, and other diseases that may cause NAFLD and those with type 1 diabetes and other particular types of diabetes.

\section{Methods}

\section{Collection of general characteristics of the study subjects}

The general information (name, sex, and age) such as diabetes duration, medication status, personal history, and any other medical history of each study participant was collected. The height, weight, and blood pressure were measured and recorded.

BMI was calculated using the following formula: BMI = weight $(\mathrm{kg}) /$ height $\left(\mathrm{m}^{2}\right)$.

\section{Laboratory examinations}

We extracted $5 \mathrm{~mL}$ of venous blood from all of the patients in the morning after $8 \mathrm{~h}$ of fasting, and the serum was separated. Serum ferritin, folic acid (FA), vitamin B12 (VB12), homocysteine (Hcy), aspartate transaminase (AST), alanine aminotransferase (ALT), direct bilirubin (DBil), total bilirubin (TBil), alkaline phosphatase (ALP), uric acid (UA), lactate dehydrogenase (LDL), total protein (TP), albumin, low-density lipoprotein cholesterol (LDL-C), total cholesterol (TC), triglyceride (TG), HDL-C, and fasting plasma glucose (FPG) were measured using automatic biochemical analyzers. Red blood cells (RBC) count, hemoglobin $(\mathrm{Hb})$ levels, and mean corpuscular $\mathrm{Hb}$ concentration (MCHC) levels were determined using a Mindray automated blood cell analyzer (Mindray Medical

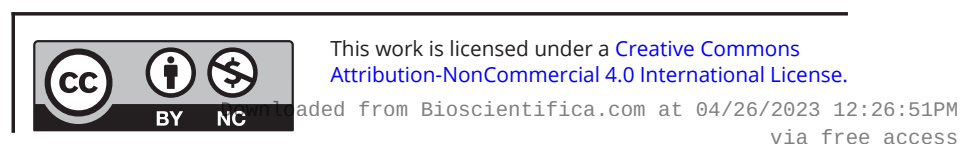


International Limited, Shenzhen, China). Glycated $\mathrm{Hb}$ (HbA1c) was determined using HPLC (Bio-Rad D10; BioRad Laboratories). Fasting insulin (FINS) was determined using the chemiluminescence method (CENTAUR-XP automat chemiluminescence immunoanalyzer; Siemens Healthineers).

The insulin resistance index (HOMA-IR) was calculated using the following formula: HOMA-IR $=F P G \times F I N S / 22.5$.

\section{Diagnostic criteria and grouping}

\section{Diagnostic criteria}

Diabetes was diagnosed according to the 1999 World Health Organization diagnostic criteria, whereas NAFLD was diagnosed according to the criteria published by the American Society of Liver Diseases in 2017. Each patient was examined using abdominal ultrasound by an experienced sonographer. Two of the following abdominal ultrasound findings suggested diffuse fatty liver: (i) the near-field echo of the liver had diffused enhancement, which was stronger than that of the kidney and spleen; (ii) the intrahepatic duct structure was not clear, and the far-field echo of the liver gradually weakened.

\section{Study population grouping}

The patients with T2DM were divided into those with and without NAFLD, and the difference in serum ferritin levels between the two groups was compared.

The patients with T2DM with NAFLD were grouped according to the quintile levels of serum ferritin: $\mathrm{F}_{1}(0-76.5$ $\mathrm{ng} / \mathrm{mL}), \mathrm{F}_{2}(76.5-117.88 \mathrm{ng} / \mathrm{mL}), \mathrm{F}_{3}(117.88-181.31 \mathrm{ng} / \mathrm{mL})$, $\mathrm{F}_{4}(181.31-265.56 \mathrm{ng} / \mathrm{mL})$, and $\mathrm{F}_{5}(\geq 265.56 \mathrm{ng} / \mathrm{mL})$. The differences in the constituent ratios of NAFLD among the five groups were compared.

\section{Statistical analysis}

All data were analyzed by SPSS version 26.0 software (IBM Corp.). Normally distributed measurement data were expressed as mean \pm S.D. $(\bar{x} \pm s)$, whereas non-normally distributed measurement data were expressed as median and interquartile range. The independent sample $t$-test was used to compare the differences of measurement data with normal distribution. The Mann-Whitney $U$-test was used to compare the differences of measurement data with nonnormal distribution. The enumeration data are expressed as frequencies and percentages (\%), and differences were compared using a chi-square test. One-way ANOVA was used to compare the differences between groups of the ordered classification data with normal distribution, whereas the
Kruskal-Wallis H test was used to compare the differences between groups of the ordered classification data with non-normal distribution. The correlation of continuous variables with non-normal distribution was analyzed by Spearman's rank-order correlation. The risk factors for NAFLD were evaluated by binary logistic regression analysis. A $P$ value of $<0.05$ was considered statistically significant.

\section{Results}

\section{Comparison of general characteristics of patients with T2DM with and without NAFLD}

Among the 805 patients with T2DM, 427 were in the NAFLD group and 378 in the non-NAFLD group. The ages, BMI levels, and diabetes duration were significantly higher in the NAFLD group than in the non-NAFLD group (Table 1).

\section{Comparison of serum ferritin and the other indices between patients with T2DM with and without NAFLD}

As shown in Table 1, levels of serum ferritin, BMI, FPG, HOMA-IR, RBC count, MCHC, Hcy, AST, ALT, DBil, TBil, ALP, UA, LDH, TP, albumin, TC, TG, and systolic blood pressure (SBP) were higher in the NAFLD group than in the non-NAFLD group $(P<0.05)$, whereas $\mathrm{Hb}, \mathrm{VB12}, \mathrm{FA}$, and HDL-C levels were lower in the NAFLD group $(P<0.05)$. However, there were no significant differences in the levels of HbA1c and LDL-C and diastolic blood pressure (DBP) levels between both groups.

\section{Comparison of the constituent ratios of NAFLD in the different ferritin levels groups}

The patients with T2DM with NAFLD were grouped according to the quintile levels of serum ferritin. As shown in Fig. 1, the NAFLD constituent ratios were significantly higher in the $\mathrm{F}_{5}$ and $\mathrm{F}_{4}$ groups than those in the $\mathrm{F}_{2}$ or $\mathrm{F}_{1}$ groups $(P<0.05)$. However, no significant difference was found between the other groups.

\section{Comparison of the biochemical indices in the different ferritin levels of patients with T2DM with NAFLD}

After the patients with T2DM with NAFLD were grouped according to the quintile levels of serum ferritin, with the increase of serum ferritin levels, the age and diabetes duration showed a decreasing trend, whereas the FPG,

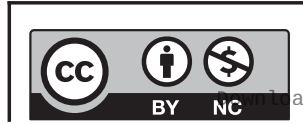

This work is licensed under a Creative Commons Attribution-NonCommercial 4.0 International License. ded from Bioscientifica.com at 04/26/2023 12:26:51PM 
Table 1 Comparison of indices between patients with T2DM with and without NAFLD. Data are presented as mean \pm S.D. or median and interquartile range.

\begin{tabular}{lc}
\hline & Non-NAFLD $(378)$ \\
\cline { 2 - 2 } \cline { 2 - 2 } Age (years) & $58.07 \pm 8.36$ \\
Duration (years) & $8.00(4.00,12.00)$ \\
BMI $\left(\mathrm{kg} / \mathrm{m}^{2}\right)$ & $23.22 \pm 2.91$ \\
Ferritin $(\mathrm{ng} / \mathrm{mL})$ & $121.19(76.97,208.39)$ \\
FPG $(\mathrm{mmol} / \mathrm{L})$ & $8.20(6.70,12.80)$ \\
HbA1c $(\%)$ & $7.95(6.80,9.80)$ \\
HOMA-IR & $2.40(1.53,3.71)$ \\
RBC $\left(10^{9} / \mathrm{L}\right)$ & $4.73 \pm 0.50$ \\
Hb $(\mathrm{g} / \mathrm{L})$ & $148.06 \pm 15.25$ \\
MCHC $(\mathrm{g} / \mathrm{L})$ & $334.00(328.00,339.00)$ \\
VB12 $(\mathrm{pg} / \mathrm{mL})$ & $399.30 \pm 145.74$ \\
FA $(\mathrm{ng} / \mathrm{mL})$ & $8.30(5.88,11.00)$ \\
Hcy $(\mu \mathrm{mol} / \mathrm{L})$ & $12.13 \pm 2.90$ \\
AST $(\mathrm{U} / \mathrm{L})$ & $19.00(16.00,22.00)$ \\
ALT $(\mathrm{U} / \mathrm{L})$ & $19.00(14.75,24.00)$ \\
DBil $(\mu \mathrm{mol} / \mathrm{L})$ & $2.70(2.10,3.30)$ \\
TBil $(\mu \mathrm{mol} / \mathrm{L})$ & $13.93 \pm 4.31$ \\
ALP $(\mathrm{U} / \mathrm{L})$ & $79.00(65.00,89.22)$ \\
UA $(\mu \mathrm{mol} / \mathrm{L})$ & $315.03 \pm 75.47$ \\
LDH $(\mathrm{U} / \mathrm{L})$ & $160.82 \pm 26.15$ \\
TP $(\mathrm{g} / \mathrm{L})$ & $68.16 \pm 6.01$ \\
Albumin $(\mathrm{g} / \mathrm{L})$ & $42.87 \pm 3.48$ \\
LDL-C $(\mathrm{mmol} / \mathrm{L})$ & $2.72 \pm 0.80$ \\
TC $(\mathrm{mmol} / \mathrm{L})$ & $4.11 \pm 0.97$ \\
TG $(\mathrm{mmol} / \mathrm{L})$ & $1.33(0.97,1.80)$ \\
HDL-C $(\mathrm{mmol} / \mathrm{L})$ & $1.03 \pm 0.21$ \\
SBP $(\mathrm{mmHg})$ & $138.23 \pm 22.69$ \\
DBP $(\mathrm{mmHg})$ & $81.98 \pm 14.65$ \\
\hline & \\
\hline
\end{tabular}

\begin{tabular}{|c|c|}
\hline NAFLD (427) & $\boldsymbol{P}$ \\
\hline $61.29 \pm 8.29$ & $<0.001$ \\
\hline $9.00(5.00,13.00)$ & 0.034 \\
\hline $25.41 \pm 2.57$ & $<0.001$ \\
\hline $168.47(103.78,248.00)$ & $<0.001$ \\
\hline $9.10(7.50,11.30)$ & $<0.001$ \\
\hline $8.30(7.10,9.80)$ & 0.062 \\
\hline $3.07(2.04,4.44)$ & $<0.001$ \\
\hline $4.82 \pm 0.57$ & 0.020 \\
\hline $145.36 \pm 15.84$ & 0.016 \\
\hline $336.00(329.00,343.00)$ & 0.001 \\
\hline $372.01 \pm 127.89$ & 0.005 \\
\hline $7.50(5.60,9.70)$ & 0.001 \\
\hline $13.24 \pm 3.47$ & $<0.001$ \\
\hline $21.00(17.00,27.00)$ & $<0.001$ \\
\hline $23.00(17.00,34.00)$ & $<0.001$ \\
\hline $2.90(2.10,3.70)$ & 0.006 \\
\hline $15.06 \pm 4.60$ & $<0.001$ \\
\hline $87.00(71.00,101.00)$ & $<0.001$ \\
\hline $338.25 \pm 72.29$ & $<0.001$ \\
\hline $169.83 \pm 27.28$ & $<0.001$ \\
\hline $69.11 \pm 5.47$ & 0.021 \\
\hline $43.86 \pm 2.94$ & $<0.001$ \\
\hline $2.76 \pm 0.71$ & 0.421 \\
\hline $4.45 \pm 0.96$ & $<0.001$ \\
\hline $1.77(1.32,2.36)$ & $<0.001$ \\
\hline $0.99 \pm 0.19$ & 0.007 \\
\hline $145.67 \pm 22.95$ & $<0.001$ \\
\hline $83.84 \pm 14.01$ & 0.067 \\
\hline
\end{tabular}

ALP, alkaline phosphatase; ALT, alanine aminotransferase; AST, aspartate aminotransferase; DBil, direct bilirubin; DBP, diastolic blood pressure; FA, folic acid; FPG, fasting blood glucose; Hb, hemoglobin; HbA1C, glycosylated hemoglobin; Hcy, homocysteine; HDL-C, high-density lipoprotein cholesterol; HOMA-IR, insulin resistance index; LDH, lactate dehydrogenase; LDL-C, low-density lipoprotein cholesterol; MCHC, mean hemoglobin concentration; RBC count, red blood cell count; SBP, systolic blood pressure; TBil, total bilirubin; TC, total cholesterol; TG, triglyceride; TP, total protein; UA, uric acid; VB12, vitamin B12.

HOMA-IR, Hb, MCHC, ALT, TBil, LDH, AST, and VB12 levels showed an increasing trend (Fig. 2).

For the $\mathrm{F}_{5}$ group, the levels of MCHC and ALT were higher than in the other four groups, HbA1c levels were higher than in the $\mathrm{F}_{4}, \mathrm{~F}_{2}$, and $\mathrm{F}_{3}$ groups, and HOMA-IR, AST, and TBil levels were higher than in the $\mathrm{F}_{1}, \mathrm{~F}_{2}$, and $\mathrm{F}_{3}$ groups, the FPG levels were higher than in the $\mathrm{F}_{1}$ and $\mathrm{F}_{2}$ groups, and $\mathrm{Hb}$ levels were higher than in the $\mathrm{F}_{1}$ group. However, the diabetes duration in the $\mathrm{F}_{5}$ group was lower than in the other four groups, and the age was lower than in the $\mathrm{F}_{2}$ and $\mathrm{F}_{1}$ groups (Table 2).

For the $\mathrm{F}_{4}$ group, the levels of AST and ALT were higher than in the $F_{2}$ and $F_{1}$ groups, HOMA-IR levels were higher than in the $\mathrm{F}_{2}$ group, $\mathrm{Hb}, \mathrm{MCHC}$, and TBil levels were higher than in the $F_{1}$ group, and the ages were lower than in the $\mathrm{F}_{1}$ group (Table 2 ).

For the $\mathrm{F}_{3}$ group, the levels of $\mathrm{HbA1c}$ were higher than in the $\mathrm{F}_{1}$ and $\mathrm{F}_{2}$ groups, and $\mathrm{Hb}, \mathrm{MCHC}$, ALT, and TBil levels were higher than in the $\mathrm{F}_{1}$ group (Table 2).

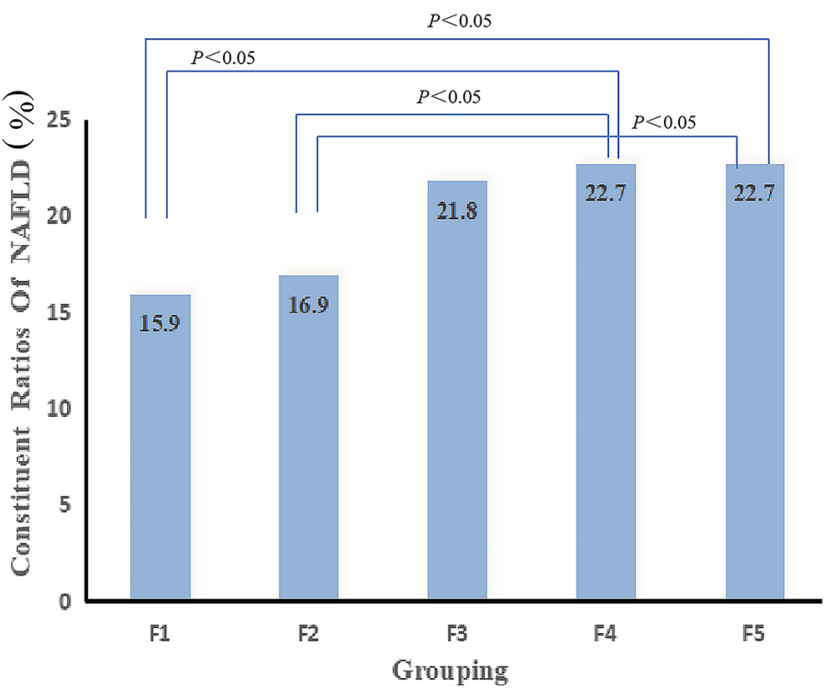

Figure 1

Comparison of the constituent ratios of NAFLD in the different ferritin level groups.

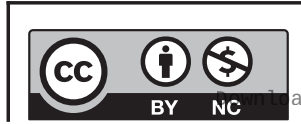

This work is licensed under a Creative Commons Attribution-NonCommercial 4.0 International License. ded from Bioscientifica.com at 04/26/2023 12:26:51PM via free access 


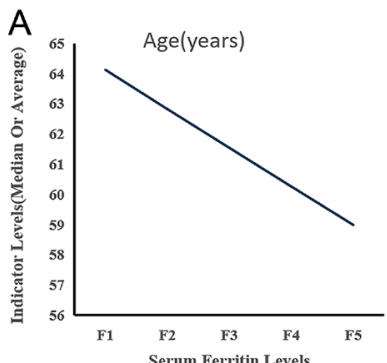

B

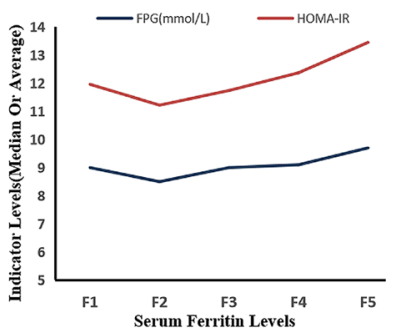

C
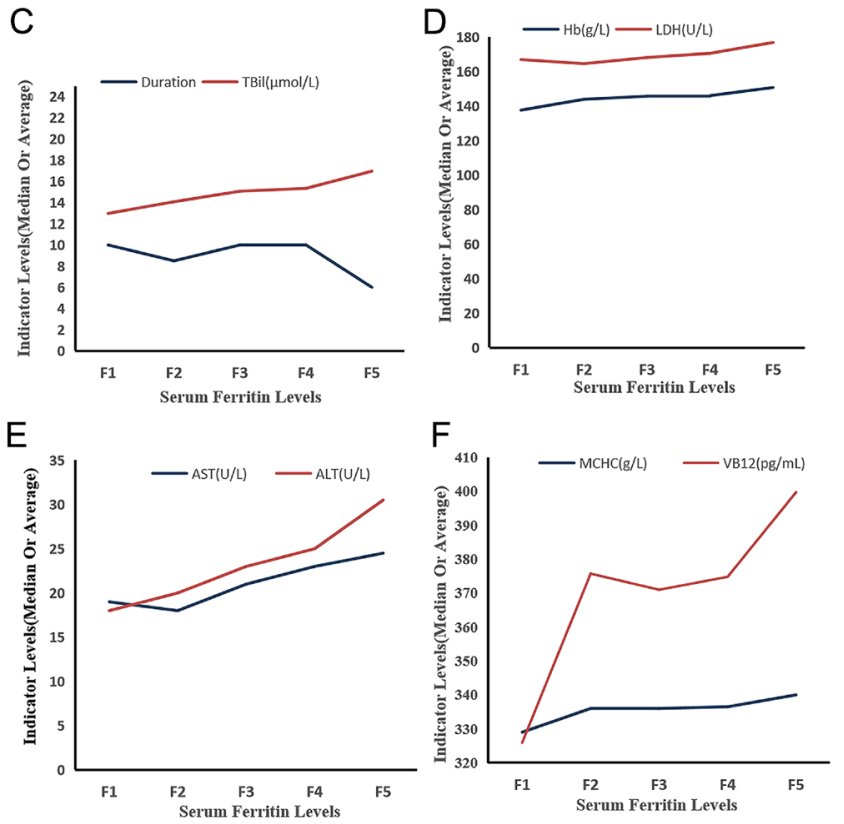

Figure 2

Comparison of the biochemical indices in different ferritin levels. (A) Age, (B) FPG, HOMA-IR, (C) duration, TBil, (D) Hb, LDH, (E) AST, ALT, and (F) $\mathrm{MCHC}, \mathrm{VB} 12$.

The levels of MCHC in the $\mathrm{F}_{2}$ group were higher than in the $\mathrm{F}_{1}$ group (Table 2 ).

\section{Correlation analysis of serum ferritin level in patients with T2DM with NAFLD}

FPG, HbA1c, HOMA-IR, Hb, MCHC, VB12, Hcy, AST, ALT, DBil, TBil, ALP, UA, LDH, RBC count, TG, TC, and DBP levels were positively correlated with serum ferritin $(P<0.05)$, whereas the age, diabetes duration, and FA levels were significantly negatively correlated with serum ferritin $(P<0.05)$ (Table 3$)$.

\section{Analysis of independent risk factors for NAFLD in patients with T2DM}

NAFLD was considered the dependent variable, and serum ferritin was the independent variable. Logistic regression analysis showed that ferritin was an independent risk factor for NAFLD $(\mathrm{OR}=1.002, P=0.001)$ (Table 4).

After adjusting for age, diabetes duration, and BMI, ferritin was still an independent risk factor for NAFLD $(\mathrm{OR}=1.002 ; P<0.05)$ (Table 4).

After further adjusting for $\mathrm{Hb}$, MCHC, RBC count, VB12, FA, AST, albumin, TBil, TP, DBil, UA, TC, HDL-C, LDH, FPG, HbA1c, SBP, DBP, ferritin, age, BMI, AST, albumin, TC, FPG, and RBC were shown to be independent risk factors for NAFLD $(\mathrm{OR}=1.002,1.064$, $1.340,1.081,1.264,1.705,1.149$, and 3.360, respectively; all $P<0.05)$. Hb, VB12, and TP were protective factors for NAFLD $(\mathrm{OR}=0.941, P<0.001 ; \mathrm{OR}=0.998, P=0.002$; and $\mathrm{OR}=0.929, P=0.001$, respectively). Diabetes duration, MCHC, FA, TBil, DBil, UA, HDL-C, LDH, SBP, HbA1c, and DBP were not associated with the risk of developing NAFLD (Table 4).

\section{Discussion}

Previous studies have shown that the level of serum ferritin, which is the main storage form of iron in the body, is correlated with T2DM and NAFLD (20, 21). Amin et al. reported that serum ferritin levels were increased in patients with T2DM with NAFLD (22). In the present study, compared with non-NAFLD T2DM patients, patients with T2DM with NAFLD had significantly increased serum ferritin levels. With the increase in serum ferritin levels, the NAFLD constituent ratios were significantly increased. Serum ferritin is an independent risk factor for NAFLD in patients with T2DM.

Studies have found that hyperferritinemia with normal transferrin saturation is a marker of glucose or lipid metabolism disorder (23). In the case of iron overload, patients have an increased incidence of T2DM, and iron chelation therapy may improve diabetes by reducing iron overload (24). Iron overload may increase the risk of diabetes through oxidative stress, inflammation, insulin resistance, insulin deficiency, liver dysfunction, and other conditions. Conversely, diabetes may also disrupt iron metabolism homeostasis and cause iron overload, forming a vicious cycle (25). In addition, liver injury is associated with hepatic iron itself. One study found that reducing consumption of foods higher in iron possibly prevented NAFLD (26). A significant relationship has been reported between iron content in the adipose tissue and adipose tissue dysfunction with the dysregulation of adipokines through lipolysis and inflammation (27). https://ec.bioscientifica.com

https://doi.org/10.1530/EC-21-0367 (c) 2021 The authors Published by Bioscientifica Ltd
This work is licensed under a Creative Commons Attribution-NonCommercial 4.0 International License. ded from Bioscientifica.com at 04/26/2023 12:26:51PM 


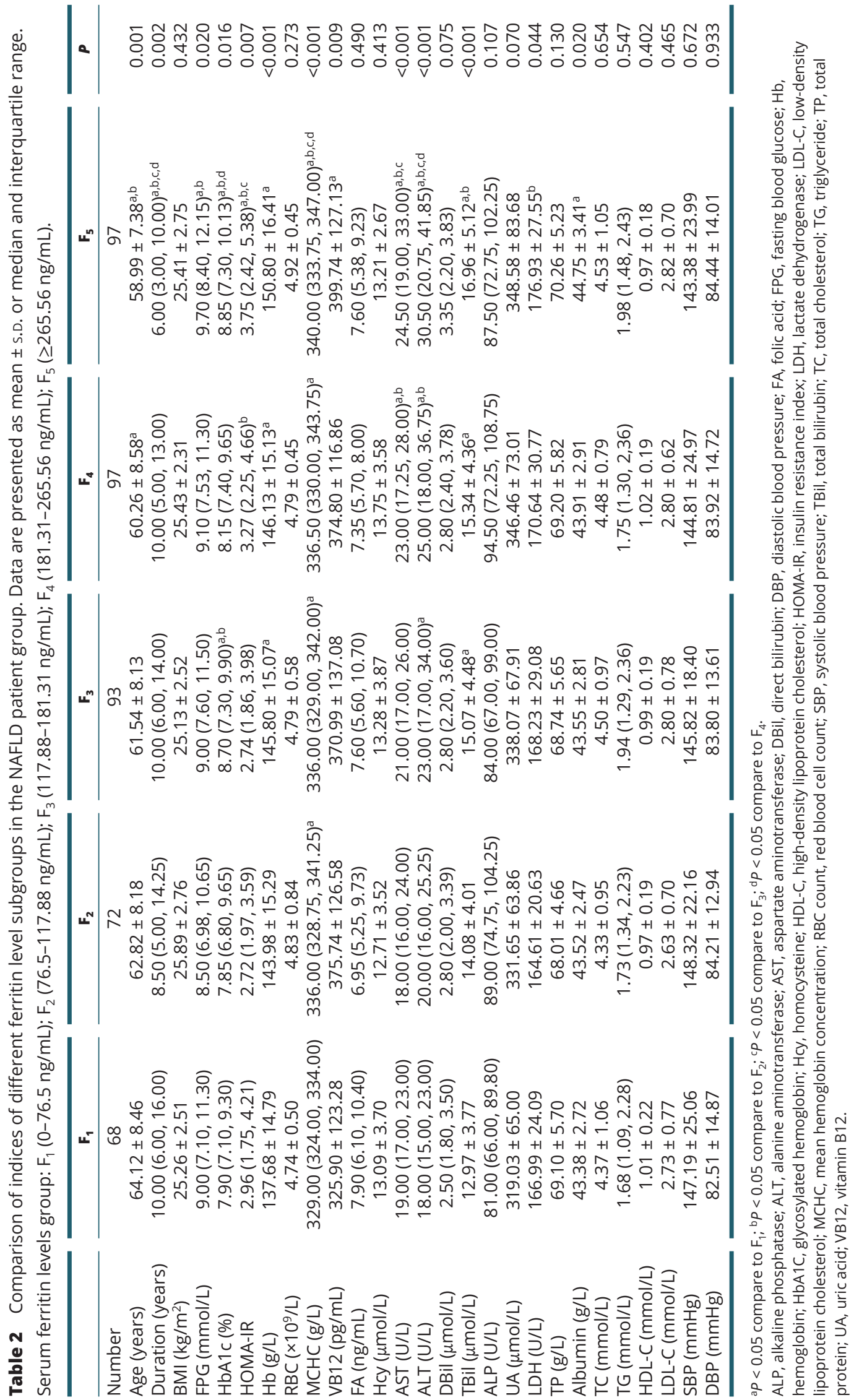


Table 3 Correlation analysis of serum ferritin levels and other indices in the NAFLD group.

\begin{tabular}{|c|c|c|}
\hline & $\mathbf{R}$ & $p$ \\
\hline Age & -0.073 & 0.040 \\
\hline Duration & -0.097 & 0.006 \\
\hline BMI & 0.066 & 0.060 \\
\hline FPG & 0.150 & $<0.001$ \\
\hline HbA1c & 0.179 & $<0.001$ \\
\hline HOMA-IR & 0.129 & $<0.001$ \\
\hline $\mathrm{Hb}$ & 0.262 & $<0.001$ \\
\hline $\mathrm{MCHC}$ & 0.284 & $<0.001$ \\
\hline VB12 & 0.159 & $<0.001$ \\
\hline FA & -0.171 & $<0.001$ \\
\hline Hcy & 0.117 & 0.001 \\
\hline AST & 0.190 & $<0.001$ \\
\hline $\mathrm{ALT}$ & 0.300 & $<0.001$ \\
\hline DBil & 0.151 & $<0.001$ \\
\hline TBil & 0.206 & $<0.001$ \\
\hline ALP & 0.104 & 0.003 \\
\hline RBC count & 0.165 & $<0.001$ \\
\hline UA & 0.145 & $<0.001$ \\
\hline $\mathrm{LDH}$ & 0.136 & $<0.001$ \\
\hline $\mathrm{TP}$ & 0.010 & 0.744 \\
\hline Albumin & 0.061 & 0.093 \\
\hline $\mathrm{TG}$ & 0.141 & $<0.001$ \\
\hline $\mathrm{TC}$ & 0.094 & 0.008 \\
\hline HDL-C & -0.057 & 0.108 \\
\hline LDL-C & 0.065 & 0.064 \\
\hline SBP & -0.015 & 0.670 \\
\hline DBP & 0.074 & 0.036 \\
\hline
\end{tabular}

ALP, alkaline phosphatase; ALT, alanine aminotransferase; AST, aspartate aminotransferase; DBil, direct bilirubin; DBP, diastolic blood pressure; FA, folic acid; FPG, fasting blood glucose; $\mathrm{Hb}$, hemoglobin; $\mathrm{HbA} 1 \mathrm{C}$, glycosylated hemoglobin; Hcy, homocysteine; HDL-C, high-density lipoprotein cholesterol; HOMA-IR, insulin resistance index; LDH, lactate dehydrogenase; LDL-C, low-density lipoprotein cholesterol; MCHC, mean hemoglobin concentration; RBC count, red blood cell count; SBP, systolic blood pressure; TBil, total bilirubin; TC, total cholesterol; TG, triglyceride; TP, total protein; UA, uric acid; VB12, vitamin B12.

Our study showed that serum ferritin levels were positively correlated with HOMA-IR. Ferritin can increase the risk of diabetes and insulin resistance through inflammation and oxidative stress (28). Insulin resistance is one of the main characteristics of NAFLD (29). In the state of insulin resistance, the $\beta$-oxidation of free fatty acids is inhibited, which further promotes the accumulation of fat in the liver (30). Mayneris-Perxachs et al. reported a positive correlation between serum ferritin levels and the degree of fat accumulation in the liver (31). There was a significant cross-talk among gut microbiota, iron status, and liver fat accumulation, and the mechanism was speculated to be microbiome- and iron-linked metabolomic and transcriptomic signatures involving imbalances in gluconeogenic metabolites, ketone bodies,
Table 4 Logistic regression analysis of influencing factors of NAFLD in patients with T2DM.

\begin{tabular}{|c|c|c|c|c|}
\hline & B & $P$ & OR & $95 \% \mathrm{Cl}$ \\
\hline \multicolumn{5}{|l|}{ Model 1} \\
\hline Ferritin & 0.002 & 0.001 & 1.002 & $1.001,1.003$ \\
\hline \multicolumn{5}{|l|}{ Model 2} \\
\hline Ferritin & 0.002 & 0.001 & 1.002 & $1.001,1.003$ \\
\hline Age & 0.052 & $<0.001$ & 1.053 & $1.053,1.074$ \\
\hline Duration & 0.022 & 0.120 & 1.022 & $0.994,1.050$ \\
\hline $\mathrm{BMI}$ & 0.292 & $<0.001$ & 1.340 & $1.262,1.422$ \\
\hline \multicolumn{5}{|l|}{ Model 3} \\
\hline Ferritin & 0.002 & 0.048 & 1.002 & $1.000,1.003$ \\
\hline Age & 0.062 & $<0.001$ & 1.064 & $1.038,1.091$ \\
\hline Duration & 0.030 & 0.070 & 1.031 & $0.998,1.065$ \\
\hline $\mathrm{BMI}$ & 0.310 & $<0.001$ & 1.363 & $1.265,1.469$ \\
\hline $\mathrm{Hb}$ & -0.061 & $<0.001$ & 0.941 & $0.920,0.962$ \\
\hline $\mathrm{MCHC}$ & 0.014 & 0.183 & 1.014 & $0.994,1.034$ \\
\hline VB12 & -0.002 & 0.002 & 0.998 & $0.996,0.999$ \\
\hline FA & -0.059 & 0.056 & 0.943 & $0.887,1.001$ \\
\hline AST & 0.078 & $<0.001$ & 1.081 & $1.045,1.119$ \\
\hline Albumin & 0.235 & $<0.001$ & 1.264 & $1.167,1.370$ \\
\hline TBil & 0.013 & 0.712 & 1.013 & $0.944,1.087$ \\
\hline $\mathrm{TP}$ & -0.073 & 0.001 & 0.929 & $0.891,0.969$ \\
\hline DBil & 0.197 & 0.215 & 1.217 & $0.892,1.661$ \\
\hline UA & 0.000 & 0.974 & 1.000 & $0.997,1.003$ \\
\hline $\mathrm{TC}$ & 0.534 & $<0.001$ & 1.705 & $1.364,2.132$ \\
\hline SBP & 0.007 & 0.237 & 1.007 & $0.994,1.009$ \\
\hline HDL-C & -0.867 & 0.099 & 0.420 & $0.150,1.178$ \\
\hline LDH & 0.002 & 0.678 & 1.002 & $0.994,1.009$ \\
\hline RBC count & 1.212 & $<0.001$ & 3.360 & $1.760,6.413$ \\
\hline $\mathrm{HbA} 1 \mathrm{c}$ & -0.012 & 0.851 & 0.989 & $0.876,1.115$ \\
\hline FPG & 0.139 & 0.001 & 1.149 & $1.058,1.247$ \\
\hline DBP & -0.011 & 0.275 & 0.990 & $0.971,1.008$ \\
\hline
\end{tabular}

Model 1, not adjusted; Model 2, adjusted age, duration, BMl; Model 3, adjusted Model $2+\mathrm{Hb}, \mathrm{MCHC}$, VB12, FA, AST, albumin, TBil, TP, DBil, UA, TC, SBP, HDL, LDH, RBC count, HbA1c, FPG, DBP.

ALP, alkaline phosphatase; ALT, alanine aminotransferase; AST, aspartate aminotransferase; DBil, direct bilirubin; DBP, diastolic blood pressure; FA, folic acid; FPG, fasting blood glucose; $\mathrm{Hb}$, hemoglobin; $\mathrm{HbA1C}$, glycosylated hemoglobin; Hcy, homocysteine; HDL-C, high-density lipoprotein cholesterol; HOMA-IR, insulin resistance index; LDH, lactate dehydrogenase; LDL-C, low-density lipoprotein cholesterol; MCHC, mean hemoglobin concentration; RBC count, red blood cell count; SBP, systolic blood pressure; TBil, total bilirubin; TC, total cholesterol; TG, triglyceride; TP, total protein; UA, uric acid; VB12, vitamin B12.

and cellular transport, which altogether modulate liver fat accumulation (31).

The incidence of NAFLD in obese people is 3.5 times that of people with normal weight (32). The overall prevalence of obesity in patients with NAFLD is approximately 51.34\% (33). Higher BMI and visceral obesity are common risk factors for NAFLD (34). In the present study, the levels of BMI of patients with T2DM with NAFLD were higher than those of T2DM without NAFLD, and BMI was shown as an independent risk factor for the occurrence of NAFLD. Obesity is closely related https://ec.bioscientifica.com

https://doi.org/10.1530/EC-21-0367 (c) 2021 The authors Published by Bioscientifica Ltd

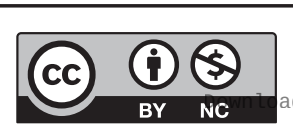

This work is licensed under a Creative Commons Attribution-NonCommercial 4.0 International License. ded from Bioscientifica.com at 04/26/2023 12:26:51PM via free access 
to metabolic dysfunction, such as insulin resistance and dyslipidemia, which further promotes the development of NAFLD (35). Meanwhile, chronic inflammation caused by excessive lipid accumulation in obese patients also plays an important role in the development of NAFLD (36).

In this study, blood glucose, especially FPG and HOMA-IR, were closely related to the occurrence of NAFLD in patients with T2DM, whereas a positive correlation between serum ferritin levels and FPG was shown in T2DM with NAFLD. Zou et al. reported that a higher FPG level was a risk factor for NAFLD (37). First, the higher FPG induces hepatocyte toxicity through oxidative stress and endoplasmic reticulum stress, leading to hepatocyte necrosis. Secondly, chronic hyperglycemia leads to metabolic disorders in the liver that promotes inflammation and insulin resistance and induces new fat formation (38). Finally, insulin resistance promotes the release of various inflammatory factors, which further increases NAFLD risk (39).

NAFLD and MS are two different entities sharing common clinical and physio-pathological features. First, NAFLD and MS have reached epidemic proportions and are the most common causes of chronic liver diseases in Western countries. Secondly, they share several common pathological features, such as insulin resistance, which is the main manifestation of both. Insulin resistance can increase hepatic lipogenesis and inhibit the lipolysis of adipose tissue, this results in an accumulation of free fatty acids (FFAs) inside the liver (FFA), which aggravates insulin resistance by inhibiting insulin receptor signaling pathway. Finally, genetic and epidemiological factors, including diet, lifestyle, mitochondrial dysfunction, lowgrade chronic inflammation, adipose tissue dysfunction, oxidative stress, microbial, and sustained immunity may also play important roles in the development of NAFLD and MS. Most importantly, MS is associated with dietary and lifestyle changes, especially unbalanced calorie intake and energy expenditure, which are equally important in the development of NAFLD $(40,41)$.

The UA may directly affect fat accumulation and cirrhosis, inhibit insulin signaling, and cause insulin resistance (42), and lead to mitochondrial oxidative stress (43). Our study found that UA levels were increased in T2DM with NAFLD. However, no independent correlation was shown between UA and the risk of NAFLD after adjusting for confounding factors, which suggested that the effect of UA may not be direct but indirect through other risk factors for NAFLD (44). This study showed that UA levels were correlated with serum ferritin, and the mechanism may be via inflammation induced by UA through the p38 mitogenactivated protein kinase, cyclooxygenase- 2 , and chemokine cyto-chemotactic protein-1 signal pathway (45).

Abnormal liver transaminase and lipid metabolism disorders are closely related to NAFLD and can predict the risks of NAFLD. Amin et al. reported that the deterioration of lipid profiles, such as an increase in TG and TC levels, and the decrease in HDL levels were associated with the incidence of NAFLD. Liver enzymes, as the main signal of liver cell injury, were significantly correlated with HOMA-IR in T2DM patients with NAFLD (22). In this study, we found that serum ferritin was also positively correlated with liver transaminase levels.

This study has some limitations. First, it had a crosssectional design. Therefore, the observed correlations between serum ferritin and NAFLD in type 2 diabetes cannot indicate a certain cause-effect relationship. Secondly, the gold standard for the diagnosis of NAFLD is liver biopsy, whereas, in this study, different physicians used ultrasound. Thus, there may be bias in the diagnosis of NAFLD. Thirdly, the lack of NAFLD stage definition did not allow the evaluation of serum ferritin difference in the various grades of NAFLD; therefore, further studies are needed to evaluate the relationship between the degree of steatosis and serum ferritin levels. Finally, patients with diabetes were treated with different types of anti-hyperglycemic drugs, and the influence of these drugs on laboratory indicators and NAFLD could not be completely excluded.

\section{Conclusion}

Serum ferritin levels were significantly increased in T2DM patients with NAFLD, and the prevalence of NAFLD in patients with T2DM increased gradually with the increase in serum ferritin levels. Thus, serum ferritin is an independent risk factor for NAFLD in patients with T2DM. Therefore, patients with T2DM with NAFLD should be concerned about changes in serum ferritin levels, and patients with T2DM with elevated serum ferritin levels should be aware of the occurrence and progression of NAFLD.

\section{Compliance with ethical standards}

All procedures performed in studies involving human participants were in accordance with the ethical standards of the institutional and with the 1964 Helsinki declaration
This work is licensed under a Creative Commons Attribution-NonCommercial 4.0 International License. ded from Bioscientifica.com at 04/26/2023 12:26:51PM via free access 
and its later amendments or comparable ethical standards. Written and oral information of the protocol was explained to them before screening, and informed consents were obtained from each eligible participant, and consent was obtained from all patients.

\section{Declaration of interest}

The authors declare that there is no conflict of interest that could be perceived as prejudicing the impartiality of the research reported.

\section{Funding}

This study was financially supported by the National Natural Science Foundation of China (No. 81960155) and Health Industry Scientific Research Project of Gansu Province (No. GSWSKY-2019-07).

\section{Data availability statement}

The datasets generated during and analyzed during the current study are not publicly available but are available from the corresponding author on reasonable request.

\section{Acknowledgements}

The authors are deeply grateful to members: Miss Junxin Yan, Miss Binjing Pan, and Mr Pingping Zhao for collecting data. Miss Junxin Yan and Dr Jingfang Liu for writing the manuscript. Dr Jingfang Liu, Dr Songbo Fu and Dr Liting Wang for designing the study.

\section{References}

1 Younossi ZM, Koenig AB, Abdelatif D, Fazel Y, Henry L \& Wymer M. Global epidemiology of nonalcoholic fatty liver disease-meta-analytic assessment of prevalence, incidence, and outcomes. Hepatology 2016 64 73-84. (https://doi.org/10.1002/hep.28431)

2 Mitra S, De A \& Chowdhury A. Epidemiology of non-alcoholic and alcoholic fatty liver diseases. Translational Gastroenterology and Hepatology 20205 16. (https://doi.org/10.21037/tgh.2019.09.08)

3 Rosselli M, Lotersztajn S, Vizzutti F, Arena U, Pinzani M \& Marra F. The metabolic syndrome and chronic liver disease. Current Pharmaceutical Design 201420 5010-5024. (https://doi.org/10.2174/1381612819666131 206111352)

4 Smith BW \& Adams LA. Nonalcoholic fatty liver disease and diabetes mellitus: pathogenesis and treatment. Nature Reviews: Endocrinology 20117 456-465. (https://doi.org/10.1038/nrendo.2011.72)

5 Eslam M, Newsome PN, Sarin SK, Anstee QM, Targher G, RomeroGomez M, Zelber-Sagi S, Wai-Sun Wong V, Dufour JF, Schattenberg JM, et al. A new definition for metabolic dysfunction-associated fatty liver disease: an international expert consensus statement. Journal of Hepatology 202073 202-209. (https://doi.org/10.1016/j. jhep.2020.03.039)

6 Sapra A \& Bhandari P. Diabetes mellitus. In StatPearls. StatPearls Publishing, 2021. (available at: http://www.ncbi.nlm.nih.gov/books/ nbk551501/)

7 Younossi ZM, Golabi P, de Avila L, Paik JM, Srishord M, Fukui N, Qiu Y, Burns L, Afendy A \& Nader F. The global epidemiology of NAFLD and NASH in patients with type 2 diabetes: a systematic review and meta-analysis. Journal of Hepatology 201971 793-801. (https://doi org/10.1016/j.jhep.2019.06.021)

8 Bril F \& Cusi K. Nonalcoholic fatty liver disease: the new complication of type 2 diabetes mellitus. Endocrinology and Metabolism Clinics of North America 201645 765-781. (https://doi.org/10.1016/j. ecl.2016.06.005)

9 Cernea S, Cahn A \& Raz I. Pharmacological management of nonalcoholic fatty liver disease in type 2 diabetes. Expert Review of Clinical Pharmacology 2017 10 535-547. (https://doi.org/10.1080/17512 433.2017.1300059)

10 Masarone M, Rosato V, Aglitti A, Bucci T, Caruso R, Salvatore T, Sasso FC, Tripodi MF \& Persico M. Liver biopsy in type 2 diabetes mellitus: steatohepatitis represents the sole feature of liver damage. PLOS ONE 201712 e0178473. (https://doi.org/10.1371/journal. pone.0178473)

11 Puntarulo S. Iron, oxidative stress and human health. Molecular Aspects of Medicine 200526 299-312. (https://doi.org/10.1016/j. mam.2005.07.001)

12 Rajpathak SN, Crandall JP, Wylie-Rosett J, Kabat GC, Rohan TE \& Hu FB. The role of iron in type 2 diabetes in humans. Biochimica et Biophysica Acta 20091790 671-681. (https://doi.org/10.1016/j. bbagen.2008.04.005)

13 Son NE. Influence of ferritin levels and inflammatory markers on HbA1c in the type 2 diabetes mellitus patients. Pakistan Journal of Medical Sciences 201935 1030-1035. (https://doi.org/10.12669/ pjms.35.4.1003)

14 Fernández-Real JM \& Manco M. Effects of iron overload on chronic metabolic diseases. Lancet: Diabetes and Endocrinology 20142 513-526. (https://doi.org/10.1016/S2213-8587(13)70174-8)

15 Valenzuela R, Rincón-Cervera MÁ, Echeverría F, Barrera C, Espinosa A, Hernández-Rodas MC, Ortiz M, Valenzuela A \& Videla LA. Ironinduced pro-oxidant and pro-lipogenic responses in relation to impaired synthesis and accretion of long-chain polyunsaturated fatty acids in rat hepatic and extrahepatic tissues. Nutrition $2018 \mathbf{4 5} 49-58$. (https://doi.org/10.1016/j.nut.2017.07.007)

16 Rolo AP, Teodoro JS \& Palmeira CM. Role of oxidative stress in the pathogenesis of nonalcoholic steatohepatitis. Free Radical Biology and Medicine 201252 59-69. (https://doi.org/10.1016/j. freeradbiomed.2011.10.003)

17 Britton L, Bridle K, Reiling J, Santrampurwala N, Wockner L, Ching H, Stuart K, Subramaniam VN, Jeffrey G, St Pierre T, et al. Hepatic iron concentration correlates with insulin sensitivity in nonalcoholic fatty liver disease. Hepatology Communications 20182 644-653. (https://doi. org/10.1002/hep4.1190)

18 Buzzetti E, Petta S, Manuguerra R, Luong TV, Cabibi D, Corradini E, Craxì A, Pinzani M, Tsochatzis E \& Pietrangelo A. Evaluating the association of serum ferritin and hepatic iron with disease severity in non-alcoholic fatty liver disease. Liver International 201939 1325-1334. (https://doi.org/10.1111/liv.14096)

19 Ryan JD, Armitage AE, Cobbold JF, Banerjee R, Borsani O, Dongiovanni P, Neubauer S, Morovat R, Wang LM, Pasricha SR, et al. Hepatic iron is the major determinant of serum ferritin in NAFLD patients. Liver International 201838 164-173. (https://doi.org/10.1111/ liv.13513)

20 You G, Ding J, Shen J, Wang Y \& Sun Y. Association between serum ferritin and non-alcoholic fatty liver disease among middle-aged and elderly Chinese with normal weight. Asia Pacific Journal of Clinical Nutrition 201928 747-753. (https://doi.org/10.6133/ apjcn.201912_28(4)0011)

21 Kunutsor SK, Apekey TA, Walley J \& Kain K. Ferritin levels and risk of type 2 diabetes mellitus: an updated systematic review and metaanalysis of prospective evidence. Diabetes/Metabolism Research and Reviews 201329 308-318. (https://doi.org/10.1002/dmrr.2394)

22 Amin RF, El Bendary AS, Ezzat SE \& Mohamed WS. Serum ferritin level, microalbuminuria and non-alcoholic fatty liver disease in type 2 https://ec.bioscientifica.com https://doi.org/10.1530/EC-21-0367 (c) 2021 The authors Published by Bioscientifica Ltd

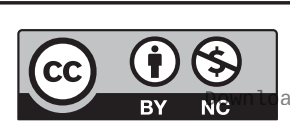

This work is licensed under a Creative Commons Attribution-NonCommercial 4.0 International License. ated from Bioscientifica com at 04/26/2023 12:26:51PM 
diabetic patients. Diabetes and Metabolic Syndrome 201913 2226-2229. (https://doi.org/10.1016/j.dsx.2019.05.030)

23 Lombardi R, Pisano G \& Fargion S. Role of serum uric acid and ferritin in the development and progression of NAFLD. International Journal of Molecular Sciences 201617 548. (https://doi.org/10.3390/ijms17040548)

24 Datz C, Felder TK, Niederseer D \& Aigner E. Iron homeostasis in the metabolic syndrome. European Journal of Clinical Investigation 201343 215-224. (https://doi.org/10.1111/eci.12032)

25 Lee HJ, Choi JS, Lee HJ, Kim WH, Park SI \& Song J. Effect of excess iron on oxidative stress and gluconeogenesis through hepcidin during mitochondrial dysfunction. Journal of Nutritional Biochemistry 201526 1414-1423. (https://doi.org/10.1016/j.jnutbio.2015.07.008)

26 Peng XE, Xu SH, Liu W, Hu Z, Lin Z \& Lin X. Independent and combined effects of dietary iron composition and selected risk factors on the risk of NAFLD in a Chinese population. Scientific Reports 20199 4069. (https://doi.org/10.1038/s41598-019-40449-1)

27 Moreno-Navarrete JM, Ortega F, Rodríguez A, Latorre J, Becerril S, Sabater-Masdeu M, Ricart W, Frühbeck G \& Fernández-Real JM. HMOX1 as a marker of iron excess-induced adipose tissue dysfunction, affecting glucose uptake and respiratory capacity in human adipocytes. Diabetologia 201760 915-926. (https://doi.org/10.1007/ s00125-017-4228-0)

28 Sachinidis A, Doumas M, Imprialos K, Stavropoulos K, Katsimardou A \& Athyros VG. Dysmetabolic iron overload in metabolic syndrome. Current Pharmaceutical Design 202026 1019-1024. (https://doi.org/10.2 174/1381612826666200130090703)

29 Park SH, Kim BI, Yun JW, Kim JW, Park DI, Cho YK, Sung IK, Park CY, Sohn CI, Jeon WK, et al. Insulin resistance and C-reactive protein as independent risk factors for non-alcoholic fatty liver disease in nonobese Asian men. Journal of Gastroenterology and Hepatology 200419 694-698. (https://doi.org/10.1111/j.1440-1746.2004.03362.x)

30 Wong VW, Hui AY, Tsang SW, Chan JL, Tse AM, Chan KF, So WY, Cheng AY, Ng WF, Wong GL, et al. Metabolic and adipokine profile of Chinese patients with nonalcoholic fatty liver disease. Clinical Gastroenterology and Hepatology 20064 1154-1161. (https://doi. org/10.1016/j.cgh.2006.06.011)

31 Mayneris-Perxachs J, Cardellini M, Hoyles L, Latorre J, Davato F, Moreno-Navarrete JM, Arnoriaga-Rodríguez M, Serino M, Abbott J, Barton RH, et al. Iron status influences non-alcoholic fatty liver disease in obesity through the gut microbiome. Microbiome 20219104. (https://doi.org/10.1186/s40168-021-01052-7)

$32 \mathrm{Li} \mathrm{L}$, Liu DW, Yan HY, Wang ZY, Zhao SH \& Wang B. Obesity is an independent risk factor for non-alcoholic fatty liver disease: evidence from a meta-analysis of 21 cohort studies. Obesity Reviews 201617 510-519. (https://doi.org/10.1111/obr.12407)

33 Saitta C, Pollicino T \& Raimondo G. Obesity and liver cancer. Annals of Hepatology 201918 810-815. (https://doi.org/10.1016/j. aohep.2019.07.004)

34 Vusirikala A, Thomas T, Bhala N, Tahrani AA, Thomas GN \& Nirantharakumar K. Impact of obesity and metabolic health status in the development of non-alcoholic fatty liver disease (NAFLD): a United Kingdom population-based cohort study using the Health
Improvement Network (THIN). BMC Endocrine Disorders 20202096. (https://doi.org/10.1186/s12902-020-00582-9)

35 Polyzos SA, Kountouras J \& Mantzoros CS. Obesity and nonalcoholic fatty liver disease: from pathophysiology to therapeutics. Metabolism: Clinical and Experimental 201992 82-97. (https://doi.org/10.1016/j. metabol.2018.11.014)

36 Tilg H, Moschen AR \& Szabo G. Interleukin-1 and inflammasomes in alcoholic liver disease/acute alcoholic hepatitis and nonalcoholic fatty liver disease/nonalcoholic steatohepatitis. Hepatology 201664 955-965. (https://doi.org/10.1002/hep.28456)

37 Zou Y, Yu M \& Sheng G. Association between fasting plasma glucose and nonalcoholic fatty liver disease in a nonobese Chinese population with normal blood lipid levels: a prospective cohort study. Lipids in Health and Disease 202019 145. (https://doi.org/10.1186/s12944-02001326-3)

38 Mota M, Banini BA, Cazanave SC \& Sanyal AJ. Molecular mechanisms of lipotoxicity and glucotoxicity in nonalcoholic fatty liver disease. Metabolism: Clinical and Experimental 201665 1049-1061. (https://doi. org/10.1016/j.metabol.2016.02.014)

39 Giovannucci E, Harlan DM, Archer MC, Bergenstal RM, Gapstur SM, Habel LA, Pollak M, Regensteiner JG \& Yee D. Diabetes and cancer: a consensus report. Diabetes Care 201033 1674-1685. (https://doi. org/10.2337/dc10-0666)

40 Rinaldi L, Pafundi PC, Galiero R, Caturano A, Morone MV, Silvestri C, Giordano M, Salvatore T \& Sasso FC. Mechanisms of non-alcoholic fatty liver disease in the metabolic syndrome. A narrative review. Antioxidants 202110 270. (https://doi.org/10.3390/antiox10020270)

41 Caturano A, Acierno C, Nevola R, Pafundi PC, Galiero R, Rinaldi L, Salvatore T, Adinolfi LE \& Sasso FC. Non-alcoholic fatty liver disease: from pathogenesis to clinical impact. Processes 20219 135. (https:// doi.org/10.3390/pr9010135)

42 Zhu Y, Hu Y, Huang T, Zhang Y, Li Z, Luo C, Luo Y, Yuan H, Hisatome I, Yamamoto T, et al. High uric acid directly inhibits insulin signalling and induces insulin resistance. Biochemical and Biophysical Research Communications 2014447 707-714. (https://doi.org/10.1016/j. bbrc.2014.04.080)

43 Lanaspa MA, Sanchez-Lozada LG, Choi YJ, Cicerchi C, Kanbay M, Roncal-Jimenez CA, Ishimoto T, Li N, Marek G, Duranay M, et al. Uric acid induces hepatic steatosis by generation of mitochondrial oxidative stress: potential role in fructose-dependent and -independent fatty liver. Journal of Biological Chemistry 2012287 40732-40744. (https://doi.org/10.1074/jbc.M112.399899)

44 Xu L, Li T, Yin J, Lin G, Xu Y, Ren Y, Wang Y, Yang J \& Chen L. Association between serum uric acid and nonalcoholic fatty liver disease in community patients with type 2 diabetes mellitus. PeerJ 20197 e7563. (https://doi.org/10.7717/peerj.7563)

45 Pallayova M, Brenisin M, Putrya A, Vrsko M, Drazilova S, Janicko M, Marekova M, Pella D, Geckova AM, Urdzik P, et al. Roma ethnicity and sex-specific associations of serum uric acid with cardiometabolic and hepatorenal health factors in eastern Slovakian population: the HepaMeta study. International Journal of Environmental Research and Public Health 202017 7673. (https://doi.org/10.3390/ijerph17207673)

Received in final form 25 October 2021

Accepted 5 November 2021

Accepted Manuscript published online 5 November 2021 https://ec.bioscientifica.com https://doi.org/10.1530/EC-21-0367 (c) 2021 The authors Published by Bioscientifica Ltd

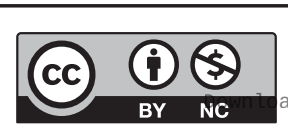

This work is licensed under a Creative Commons Attribution-NonCommercial 4.0 International License. ded from Bioscientifica com at 04/26/2023 12:26:51PM 